\title{
Recension: \\ Doris Angst / Emma Lantschner (Hrsg.), ICERD, Internationales Überein- kommen zur Beseitigung jeder Form von Rassendiskriminierung,
} Handkommentar

Rédigé par une trentaine d'experts provenant d'Allemagne, d'Autriche, du Liechtenstein et de la Suisse, ce commentaire de la Convention internationale de 1965 sur l'élimination de toutes les formes de discrimination raciale se révèle remarquable à plus d'un titre. Par son ampleur d'abord: en quelque 700 pages, cet ouvrage détaille les tenants et aboutissants de cet instrument fondateur de la lutte contre le racisme. Par sa démarche originale ensuite : à l'analyse proprement juridique de chacune des dispositions, s'ajoutent de précieux considérants sur leur contexte social et politique, ainsi que sur leur rapport aux autres instruments, notamment européens, destinés à combattre la discrimination. Par son actualité enfin: en ces temps de forte résurgence de l'intolérance envers «l'autre» et de multiplication des agressions à connotation raciste, l'ICERD, que la Suisse a ratifiée il y a vingt-cinq ans, est plus que jamais indispensable.

I. Introduction

II. L'ICERD

III. Le commentaire Angst/Lantschner

IV. Évaluation globale

Citation:

BERTIL COTTIER, Recension: Doris Angst / Emma Lantschner (Hrsg.), ICERD, Internationales Übereinkommen zur Beseitigung jeder Form von Rassendiskriminierung, Handkommentar, sui generis 2021, p. 45

Bertil Cottier, professeur honoraire, Université de Lausanne, membre pour la Suisse de la Commission européenne contre le racisme et l'intolérance (Conseil de l'Europe) (bertil.cottier@usi.ch). URL: sui-generis.ch/167 


\section{Introduction}

1 Àl'heure où s'intensifient les discours de haine et les violences raciales, les instruments juridiques destinés à combattre la discrimination sont de plus en plus sollicités. Autant dire que le présent commentaire du plus fondamental de ces instruments, la Convention internationale de 1965 sur l'élimination de toutes les formes de discrimination raciale ${ }^{1}$ (plus connue sous son acronyme anglais ICERD), vient à point nommé. Dressant un vaste état des lieux de ce pilier du dispositif onusien de protection des droits de l'homme, il prend place au premier rang des travaux scientifiques qui ont été consacrés à l'ICERD, aux côtés des deux seuls commentaires jusqu'alors disponibles - ceux, en anglais, de Natan Lerner (3 3 ème éd, Leiden 2015) et Patrick Thornberry (Oxford 2016) - et de l'ouvrage collectif édité par David Keane et Annapurna Waughray à l'occasion du cinquantenaire de ladite convention (Manchester 2017).

\section{L'ICERD}

2 Née dans un contexte marqué d'un côté par la mémoire de la tragédie de l'Holocauste, de l'autre par la décolonisation et la lutte contre l'apartheid, l'ICERD a été ratifiée par plus de 180 États (dont la Suisse en 1994). Ceux-ci se sont notamment engagés à sanctionner pénalement le discours de haine, à bannir les mouvements racistes, à promouvoir l'égalité et à mettre un terme aux pratiques discriminatoires dans les divers secteurs de la vie courante (emploi, éducation, logement notamment). La force de l'ICERD ne tient pas uniquement aux mesures drastiques qu'elle impose, mais aussi et surtout, à l'institution d'un organisme de surveillance: le Comité pour l'élimination de la discrimination raciale (CERD).

3 À cet organe composé de dix-huit experts indépendants a été confié la triple tâche d'établir des «recommandations générales » sur la mise en œuvre des dispositions de l'ICERD (ainsi l'art. 4 qui prohibe le discours de haine et la propagande raciste a déjà fait l'objet de quatre recommandations tendantà en délimiter plus concrètement les contours, notamment au regard de la liberté d'expression), d'examiner périodiquement le respect par chaque État partie des obligations qui lui incombe (attendu pour 2020, le dixième rapport sur la Suisse a pris du retard en raison de la pandémie de Covid-19) et enfin de se prononcer sur les plaintes contre un État membre émanant de particuliers victimes de discriminations («communications individuelles»). Au demeurant, cette voie, qui débouche sur une «opinion» (non contraignante) de la

1 Convention internationale sur l'élimination de toutes les formes de discrimination raciale du 21 décembre 1965 (ICERD; RS 0.104).
CERD, n'est ouverte qu'à l'encontre des États qui ont expressément déclaré s'y soumettre (à ce jour près de soixante pays, dont la Suisse depuis 2003).

\section{Le commentaire Angst/Lantschner}

L'un des atouts du présent commentaire est justement la 4 mise en valeur du travail accompli par le CERD depuis sa session constitutive en 1970, un travail méconnu au-delà du cercle étroit des spécialistes de la matière. C'est ainsi que les auteurs non seulement ont systématiquement analysé la portée de chacun des vingt-cinq articles de l'ICERD à la lumière des nombreuses recommandations et opinions de l'organe de surveillance, mais encore ils ont établi un tableau récapitulatif de toutes les communications individuelles, relevant à chaque fois les dispositions précises qui étaient en jeu. Tout aussi instructive, notamment en raison d'utiles résumés des affaires les plus marquantes, est l'étude de l'évolution de la jurisprudence du CERD qui accompagne ce tableau. Et les auteurs de souligner, à plusieurs reprises, l'importance attachée par l'organe de surveillance à une interprétation dynamique des dispositions de l'ICERD, qu'il qualifie de «living instrument».

Le remarquable souci des auteurs de dépasser le pur 5 commentaire pour éclairer le contexte social et politique del'ICERD doit être relevé. Ainsi, après avoir retracél'historique de cet instrument, ils portent une attention soutenue aux nouveaux défis de la lutte contre le racisme (montée de la xénophobie et de l'islamophobie en particulier) et aux débats dogmatiques contemporains, telle l'opposition entre les «éliminativistes» qui prônent l'abandon de toute référence à la race-cette notion s'étant révélée dépourvue de toute pertinence scientifique - , et les «constructivistes» qui souhaitent son maintien afin de garantir une application cohérente et efficace des textes juridiques qui, comme l'ICERD, se basent sur elle. Dans la même veine de contextualisation, les auteurs examinent dans le détail l'impact de l'ICERD dans les pays germanophones, dénonçant les diverses lacunes de chacun de ces ordres juridiques. S'agissant de la Suisse, la non-prise en compte de la motivation raciste en tant que circonstance aggravante d'une infraction et l'absence de toute législation civile générale contre la discrimination sont certainement les manquements les plus criants; à quoi s'ajoutent les obstacles mis à la création d'une institution de défense des droits de l'homme, indépendante et dotée de pouvoirs décisionnels.

On saluera en outre la volonté des auteurs de confronter 6 l'ICERD aux autres instruments internationaux de même vocation (notamment la directive de l'Union européenne 
2000/43 sur l'égalité de traitement)² et de montrer leur influence bénéfique sur la jurisprudence de la CERD. Méritoire est aussi l'accent qu'ils ont mis sur les efforts déployés par les institutions du Conseil de l'Europe, à commencer par la Commission européenne contre le racisme et l'intolérance (ECRI). Une section entière de leur ouvrage est en effet consacrée à la présentation fouillée des activités de cet organe dont les prononcés (recommandations générales ou rapports de monitoring par pays) s'inspirent souvent des travaux du CERD (ce qui n'est guère étonnant si l'on sait que certains membres de l'ECRI sont passés par le CERD...); cela dit, ces deux organes diffèrent foncièrement quant à leur modus operandi : alors que le CERD agit à distance, depuis son siège de Genève, l'ECRI privilégie les rencontres sur le terrain (visites dites de contact dans les pays membres, dialogues sur place avec les représentants de la société civile et les responsables de l'administration publique).

\section{IV. Évaluation globale}

7 Enfin, on soulignera que le présent commentaire ne se borne pas à décrire. Très souvent il critique ou prend parti ; l'analyse de chacune des dispositions de l'ICERD

2 Directive 2000/43/CE du conseil du 29juin 2000 relative à la mise en œuvre du principe de l'égalité de traitement entre les personnes sans distinction de race ou d'origine ethnique se conclut en effet par un bilan intermédiaire, sous la forme d'une section finale opportunément appelée «Würdigung». Certes, on peut ne pas toujours être d'accord avec les avis exprimés (personnellement je doute que le droit européen, peu importe qu'il émane du Conseil de l'Europe ou de l'Union européenne, soit à lui seul en mesure de faire cesser les dérives haineuses sur des réseaux sociaux mondialisés). Reste que l'on doit admettre que les jugements des auteurs sont toujours le fruit d'une réflexion approfondie, étayée et pondérée. On n'en attendait pas moins de la trentaine de personnalités qui ont contribué à la réalisation de cet ouvrage de référence. Allemands, autrichiens ou suisses, ils sont tous des experts renommés. À témoin le parcours professionnel des deux conceptrices du commentaire: l'une, Doris Angst, a été pendant vingt ans secrétaire générale de la Commission fédérale contre le racisme, l'autre Emma Lantschner, professeure à l'Université de Graz, conseille depuis longtemps le Conseil de l'Europe et l'OSCE en matière de protection des minorités et de non-discrimination.
Ouvragerecensé:

«ICERD, Internationales Übereinkommen zur Beseitigung jeder Form von Rassendiskriminierung, Handkommentar», Doris Angst / Emma Lantschner (Hrsg.), Nomos Verlag, Baden-Baden 2020, ISBN: 978-3-8487-4595-1 (gedruckte Ausgabe, Hardcover) 